\title{
Chaotic behavior in the flow along a wedge modeled by the Blasius equation
}

\author{
B. Basu ${ }^{1}$, E. Foufoula-Georgiou ${ }^{1}$, and A. S. Sharma ${ }^{2}$ \\ ${ }^{1}$ Department of Civil Engineering, University of Minnesota, Minneapolis, MN 55414, USA \\ ${ }^{2}$ Department of Astronomy, University of Maryland, College Park, MD 20742, USA
}

Received: 1 December 2010 - Accepted: 25 January 2011 - Published: 8 March 2011

\begin{abstract}
The Blasius equation describes the properties of steady-state two dimensional boundary layer forming over a semi-infinite plate parallel to a unidirectional flow field. The flow is governed by a modified Blasius equation when the surface is aligned along the flow. In this paper, we demonstrate using numerical solution, that as the wedge angle increases, bifurcation occurs in the nonlinear Blasius equation and the dynamics becomes chaotic leading to nonconvergence of the solution once the angle exceeds a critical value of $22^{\circ}$. This critical value is found to be in agreement with experimental results showing the development of shock waves in the medium and also with analytical results showing multiple solutions for wedge angles exceeding a critical value. Finally, we provide a derivation of the equation governing the boundary layer flow for wedge angles exceeding the critical angle at the onset of chaos.
\end{abstract}

\section{Introduction}

The Blasius equation is used to model the boundary layer growth over a surface when the flow field is slender in nature, and is derived from the two-dimensional Navier-Stokes equation. The equation was first derived by Blasius and is given by:

$f^{\prime \prime \prime}(\eta)+f(\eta) f^{\prime \prime}(\eta)=0$

with boundary conditions:

$f(0)=a ; f^{\prime}(0)=b ; f^{\prime}(\infty)=c$

The original problem for the Blasius equation is associated with $a=b=0, c=1$, and a theoretical analysis was given

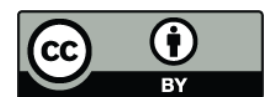

Correspondence to: B. Basu

(bidrohabasu@gmail.com) by Weyl (1942). This equation has been studied for many different conditions. Klemp and Acrivos (1972) considered $b$ being negative for a moving plate. Hussaini et al. (1986) proved and analyzed the existence and non-uniqueness of the moving plate problem. Schliching and Bussmann (1943) discussed mass transfer including injection and suction for fixed flat plate where the boundary conditions become $a \neq 0$, $b=0, c=1$. Vajravelu and Mohapatra (1990) extended the moving plate problem with mass injection on the wall to study the drag reduction effects, where $a<0, b<0, c=1$. A general discussion of this moving wall boundary layer flow was reported by Fang (2003a), where $a, b \in \Re, c=1$ ( $\Re$ is any real number). Fang (2003b) also analyzed the heat transfer problem for a moving wall boundary layer. Cortell (2007) studied the heat transfer problem for a moving impermeable wall. Another class of boundary layer problem for a stretching sheet relevant to the Blasius equation was studied by Sakiadis (1961), in which the boundary conditions become $b=1, c=0$ with $a=0$ for an impermeable plate and $a \neq 0$ for mass transfer across a permeable plate, where the $\mathrm{x}$-velocity was normalized by the wall stretching velocity. It was shown that for $a, b \in \Re, c \leq b$, the Blasius equation has one and only one solution if $b \geq 0,0 \leq c \leq b$, and no solution if $c<0$. In the real physical world, a problem for a semi-infinite flat wall moving in a stationary fluid frequently occurs. This problem can be modeled by the Blasius equation with the above-mentioned boundary conditions. Kewley and Hornung (1974) performed an experiment on a wedge of length $14 \mathrm{~cm}$ and width of $15 \mathrm{~cm}$ with wedge angle $2 \theta$. As they varied the angle of the wedge, the flow-field started to produce shock curvature at $2 \theta=35^{\circ}$ and began to create straight shock waves when the angle reached $2 \theta=43^{\circ}$. These observations of shock waves and non uniqueness in the analytical analysis motivated us to perform a nonlinear analysis on the Blasius equation for different wedge angles.

Published by Copernicus Publications on behalf of the European Geosciences Union and the American Geophysical Union. 
In the next section we sketch the derivation of the Blasius equation from the two-dimensional Navier-Stokes equation and we establish the terminology. In Sect. 3 we present the results of our numerical simulation, demonstrate the onset of chaos and quantify the angle at which the system becomes chaotic. We also provide in Sect. 4 a derivation of the equation governing the boundary layer flow for wedge angles exceeding the critical angle at the onset of chaos. Finally, conclusions are presented in Sect. 5. Details of the numerical technique are given in the Appendix A.

\section{Theory of flow along a wedge}

\subsection{Derivation of Blasius equation from Navier-Stokes equation}

Consider a uniform flow of velocity $U$ approaching a slender wedge (see Fig. 1). For a slender wedge, the equation for the flow can be derived from the two-dimensional Navier-Stokes equation:

$$
\begin{aligned}
& \frac{\partial u}{\partial x}+\frac{\partial v}{\partial y}=0 \\
& \frac{\partial u}{\partial t}+u \frac{\partial u}{\partial x}+v \frac{\partial u}{\partial y}=-\frac{1}{\rho} \frac{\partial p}{\partial x}+v\left(\frac{\partial^{2} u}{\partial x^{2}}+\frac{\partial^{2} u}{\partial y^{2}}\right) \\
& \frac{\partial v}{\partial t}+u \frac{\partial v}{\partial x}+v \frac{\partial v}{\partial y}=-\frac{1}{\rho} \frac{\partial p}{\partial y}+v\left(\frac{\partial^{2} v}{\partial x^{2}}+\frac{\partial^{2} v}{\partial y^{2}}\right)
\end{aligned}
$$

where $u$ and $v$ are the velocity components of flow in the $\mathrm{x}$ and $\mathrm{y}$ directions, respectively, $p$ is the dynamic pressure at any location $(x, y)$ and $v$ is the kinematic viscosity of the flowing fluid.

It has been observed (Schlichting and Gersten, 1999) that the velocities $u$ and $v$ are of the order of the free stream velocity $U$, except in the region close to the surface of the body. The velocity of the fluid at the surface of the wedge is zero due to no-slip condition and there is a transition from zero to full velocity at a distance from the surface. The transition occurs within a thin boundary layer where the velocity gradient $\frac{\partial u}{\partial y}$ is very large, hence the viscous shear stress $\tau=\mu \frac{\partial u}{\partial y}$ plays an important role in the flow. In the region outside the boundary layer, the velocity gradient is very small and hence the viscosity is significantly less important and the flow is frictionless.

The presence of the wedge in the flow leads to a boundary layer, as depicted in Fig. 2. At a point located at a distance $L$ along $x$ from the edge of the plate, the depth of the boundary layer is assumed to be $\delta$. We can scale the variables as $x \sim L$, $y \sim \delta ; u \sim U, v \sim V$ and $t \sim L / U$ to perform a dimensional analysis.

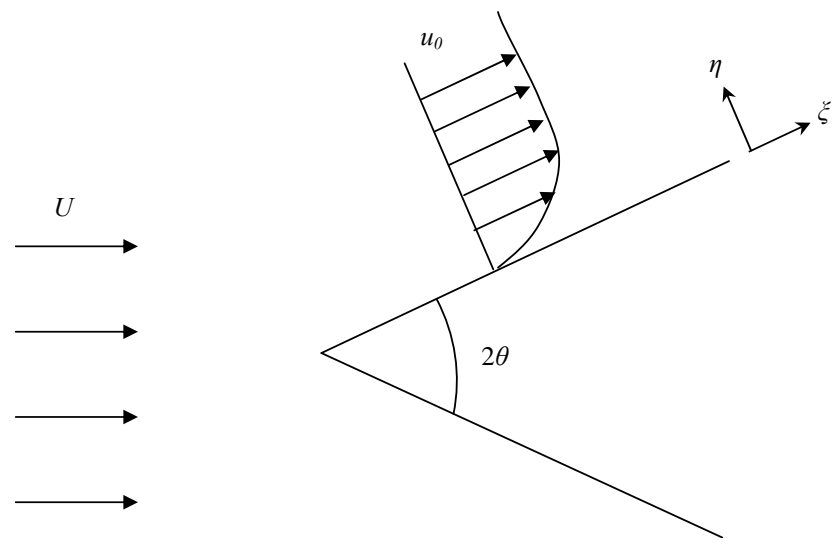

Fig. 1. Laminar boundary layer growth over a wedge of angle $2 \theta$.

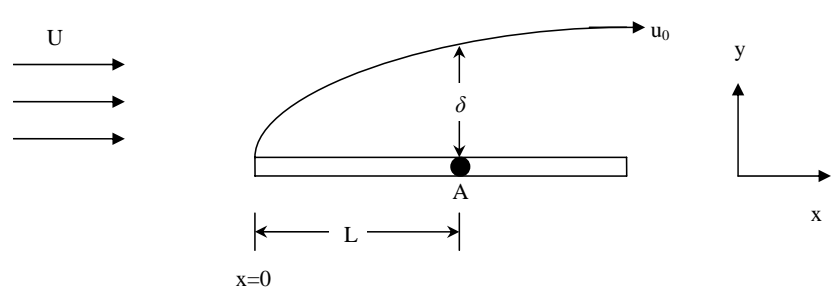

Fig. 2. Laminar boundary layer growth over a flat surface where the boundary layer depth is of the order of $\delta$ and the velocity of flow in the $\mathrm{x}$-direction at the boundary layer is $u_{0}$.

From the continuity equation, Eq. (2.1), we get $V \sim U \delta / L$. With $\frac{v}{L U} \approx \varepsilon \ll 1$, the balance between viscosity and convective inertia implies $\frac{\delta^{2}}{L^{2}} \approx \varepsilon$, and the term $v \frac{\partial^{2} u}{\partial x^{2}}$ in the $\mathrm{x}$ component of the Navier-Stokes equation, Eq. (2.2), can be neglected. Then the equation in the $\mathrm{x}$-direction becomes:

$\frac{\partial u}{\partial t}+u \frac{\partial u}{\partial x}+v \frac{\partial u}{\partial y}=-\frac{1}{\rho} \frac{\partial p}{\partial x}+v \frac{\partial^{2} u}{\partial y^{2}}$

Similarly, scaling arguments reduce the y-component of the Navier-Stokes equation, Eq. (2.3), to

$-\frac{1}{\rho} \frac{\partial p}{\partial y}=0$

At the outer boundary layer, i.e., in the inviscid region, the velocity in the $y$-direction becomes zero and the velocity in the $\mathrm{x}$-direction does not change with respect to $y$, i.e.,

$\frac{\partial u}{\partial y}=0, v=0$

For a steady state flow, $\frac{\partial u}{\partial t}=0$, and the momentum equation in the $\mathrm{x}$-direction at the boundary becomes:

$u_{0} \frac{\partial u_{0}}{\partial x}=-\frac{1}{\rho} \frac{\partial p}{\partial x}$,

where $u_{0}$ is the velocity of flow in the x-direction at the 
boundary. Then the complete set of equations consists of Eq. (2.1) and

$u \frac{\partial u}{\partial x}+v \frac{\partial u}{\partial y}=u_{0} \frac{\partial u_{0}}{\partial x}+v \frac{\partial^{2} u}{\partial y^{2}}$

$\frac{\partial p}{\partial y}=0$

To solve this system of equations the velocities are represented by the stream-function $\psi(x, y)$ of the flow defined by:

$u=\frac{\partial \psi}{\partial y}, v=-\frac{\partial \psi}{\partial x}$

Replacing velocities by the stream-function in the momentum equation gives:

$\frac{\partial \psi}{\partial y} \frac{\partial^{2} \psi}{\partial x \partial y}-\frac{\partial \psi}{\partial x} \frac{\partial^{2} \psi}{\partial y^{2}}=u_{0} \frac{\partial u_{0}}{\partial x}+v \frac{\partial^{3} \psi}{\partial y^{3}}$

The equation is made non-dimensional by defining the variables:

$\xi=\frac{x}{L}, \eta=\frac{y}{L} \frac{\sqrt{R e}}{\delta(\xi)}$

where the Reynolds number is given by $R e=\frac{U L}{v}, L$ being the characteristic length scale along $x$. Taking as a trial solution for the stream-function (Schlichting and Gersten, 1999) the form:

$\psi(\xi, \eta)=\frac{L u_{0}(\xi)}{\sqrt{\operatorname{Re}}} \delta(\xi) f(\xi, \eta)$

we get,

$\frac{u(\xi, \eta)}{u_{0}(\xi)}=f_{\eta}(\xi, \eta)$

The function $f$ is assigned in such a way that it provides a self-similar solution. Hence it becomes a function of $\eta$ only. Then replacing $\psi(x, y)$ in terms of $f(\eta)$ in the momentum equation yields, after extensive but straightforward manipulations,

$f_{\eta \eta \eta}+a_{1} f f_{\eta \eta}+a_{2}-a_{3}\left(f_{\eta}\right)^{2}=\frac{\delta^{2} u_{0}}{U} \cdot\left[f_{\eta} f_{\xi \eta}-f_{\eta \eta} f_{\xi}\right]$

where $a_{1}=\frac{\delta}{U} \frac{d}{d \xi}\left(u_{0} \delta\right), a_{2}=a_{3}=\frac{\delta^{2}}{U} \frac{d u_{0}}{d \xi}$, and the subscripts indicate partial derivatives.

If the coefficients $a_{1}, a_{2}$ and $a_{3}$ are constants, the solution $f$ is independent of $\xi$ and the equation reduces to:

$f^{\prime \prime \prime}(\eta)+a_{1} f(\eta) f^{\prime \prime}(\eta)+a_{2}-a_{3} f^{\prime}(\eta)^{2}=0$

and the variables $\xi=\frac{x}{L}, \eta=\frac{y}{L} \frac{\sqrt{R e}}{\delta(\xi)}$ become the characteristic variables of the partial differential equation. Equation (2.11) is a form of Blasius equation (Schlichting and Gersten, 1999), the common or simplified form of which corresponds to the case $a_{1}=1 / 2, a_{2}=a_{3}=0$.

\subsection{Flow over a wedge}

For a flow over a wedge, the inviscid flow velocity $u_{0}$ is assumed to follow a power law dependence with distance

$u_{0}=U x^{m}$

where $m=\beta /(2-\beta) ; \beta=2 \theta / \pi$ (see Fig. 1). This assumption is made based on different experimental observations (Schwartz and Eley, 2002).

The coefficients and the boundary conditions in the Blasius equation depend upon the values of the wedge angles. When the value of $m$ is $0 \leq m<\infty ; 0 \leq \beta<2$, then the coefficients become: $a_{1}=1, a_{2}=a_{3}=\frac{\overline{2 m}}{m+1}$ where $\eta=\frac{y}{\delta}$; $\delta=\sqrt{\frac{2 U x}{(m+1) L u_{0}}}$. In this case, the final form of the equation is written as:

$f^{\prime \prime \prime}+f f^{\prime \prime}+\frac{2 m}{m+1}\left(1-f^{2}\right)=0$
$\Rightarrow \frac{m+1}{2} f^{\prime \prime \prime}+\frac{m+1}{2} f f^{\prime \prime}+m\left(1-f^{\prime 2}\right)=0$,

where $\eta=\frac{y}{\delta} ; \delta=\sqrt{\frac{2 v x}{(m+1) u_{0}}}$,

$f^{\prime}=\frac{d f}{d \eta}=\frac{u}{u_{0}}, f^{\prime \prime}=\frac{d^{2} f}{d \eta^{2}}, f^{\prime \prime \prime}=\frac{d^{3} f}{d \eta^{3}}$

The boundary conditions are $f(0)=0 ; f^{\prime}(0)=0 ; f^{\prime}\left(\eta_{L}\right)=$ $1 ; \eta_{L}$ is a large value of $\eta$ where the velocity becomes almost laminar.

For a reversed wedge flow for which $-0.5 \leq m \leq 0 ;-2 \leq$ $\beta \leq 0$, the coefficients are (Schlichting and Gersten, 1999): $a_{1}=-1, a_{2}=a_{3}=-\frac{2 m}{m+1}$

Hence the final form of the equation becomes:

$$
\begin{aligned}
& f^{\prime \prime \prime}-f f^{\prime \prime}-\frac{2 m}{m+1}\left(1-f^{2}\right)=0 \\
& \Rightarrow \frac{m+1}{2} f^{\prime \prime \prime}-\frac{m+1}{2} f f^{\prime \prime}-m\left(1-f^{\prime 2}\right)=0
\end{aligned}
$$

along with the boundary conditions: $f(0)=0 ; f^{\prime}(0)=0$; $f^{\prime}\left(\eta_{L}\right)=1$.

Another form of the equation can be obtained by using a transformation $u=u-u_{0}$ (Brighi and Hoernel, 2006). Then the coefficients become: $a_{1}=\frac{m+1}{2} 1, a_{2}=0, a_{3}=m$. In this case, the equation takes the form (Brighi and Hoernel, 2005):

$f^{\prime \prime \prime}+\frac{m+1}{2} f f^{\prime \prime}-m f^{\prime 2}=0$

with $m=\frac{\beta+2}{2-\beta}$, so $0 \leq m<\infty,-2 \leq \beta<2$, where the boundary conditions are given by: $f(0)=$ constant; $f^{\prime}(0)=-1$; $f^{\prime}\left(\eta_{L}\right)=0$. The value of $f(0)$ is related to the value of the stream-function at the wedge surface and it depends on the assumptions used to derive the Blasius equation. It may be 
noted that for $m=0$, Eq. (2.15) reduces to the well known form of the Blasius equation.

As all the forms of the Blasius equation are third order ordinary differential equations (ODE), it is convenient to split it in three first order ODE's before solving. With $f^{\prime}=g$ and $f^{\prime \prime}=g^{\prime}=h$, the Blasius equation becomes:

$$
\begin{aligned}
& \frac{d f}{d \eta}=g \\
& \frac{d g}{d \eta}=h
\end{aligned}
$$

(Case I) $\frac{m+1}{2} \frac{d h}{d \eta}+\frac{m+1}{2} f h+m\left(1-g^{2}\right)$

$$
=0(0 \leq m<\infty ; 0 \leq \beta<2) \text { or }
$$

$$
\begin{aligned}
& \text { (Case II) } \frac{m+1}{2} \frac{d h}{d \eta}-\frac{m+1}{2} f h-m\left(1-g^{2}\right) \\
&=0(-0.5 \leq m \leq 0 ;-2 \leq \beta \leq 0) \quad \text { or }
\end{aligned}
$$

$$
\text { (Case III) } \begin{aligned}
& \frac{d h}{d \eta}+\frac{m+1}{2} f h-m g^{2} \\
& =0(0 \leq m<\infty,-2 \leq \beta<2)
\end{aligned}
$$

with proper boundary conditions.

The nonlinear differential equation can be solved by using different approaches (Brighi and Hoernel, 2005; Zaturska and Banks, 2001). One common method is to convert the boundary value problem to an initial value problem, where the value of the velocity gradient $f^{\prime \prime}(0)=h(0)=s$ is estimated by using a shooting method for which $f$ exists for $[0, \infty)$ and $f^{\prime}\left(\eta_{L}\right)=g\left(\eta_{L}\right)=1 ; \eta_{L}$ is large value of $\eta$. The usual approach to solve for $s$ is by using the NewtonRaphson method (see Appendix A).

The solution of the Blasius equation $f^{\prime \prime \prime}+\frac{m+1}{2} f f^{\prime \prime}-$ $m f^{\prime 2}=0$ where $m=\frac{\beta+2}{2-\beta}$, so $0 \leq m<\infty,-2 \leq \beta<2$ with the boundary conditions $f(0)=a ; f^{\prime}(0)=-1 ; f^{\prime}\left(\eta_{L}\right)=0$ has been obtained (Brighi and Hoernel, 2006; Brighi and Sari, 2005) by using the similarity solutions. They have observed that when $m \in[0,1]$, i.e., $-2 \leq \beta \leq 0$, there exists a unique solution for the Blasius equation for any value if $f(0)=a$. When $m>1$, i.e., $\beta>0$, there exists one unique concave solution and infinitely many concave-convex solutions for any value of $f(0)=a$.

Another approach to solving the boundary value problem is by using the Adomian decomposition method (ADM) (Adomian, 1988, 1991, 1994). In this method, the nonlinear equation is split into two components, one is the linear part and the other is the nonlinear part. The solution is then assumed to have an analytic form, given by a summation of infinite series (Allan and Syam, 2005). The coefficients of the series are then estimated by using the boundary conditions.

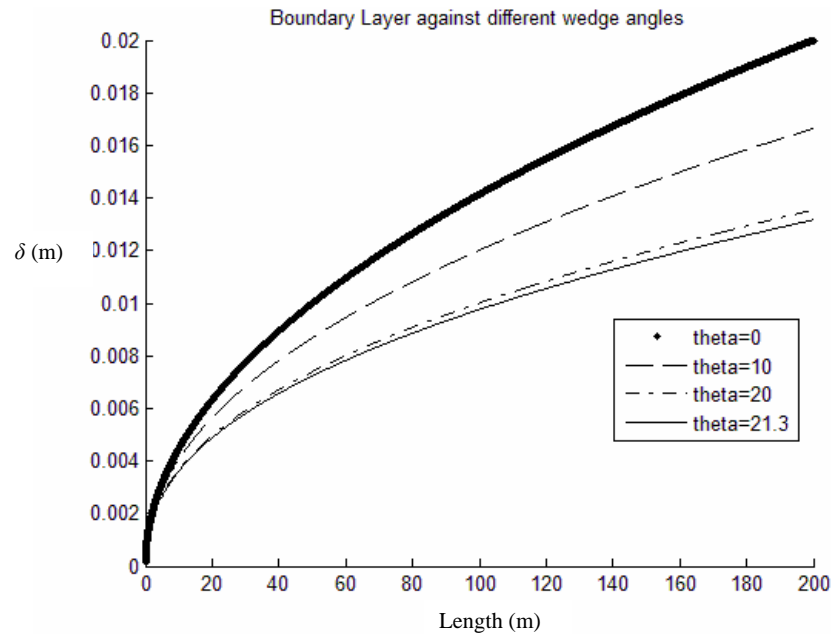

Fig. 3. The boundary layer depth against the length of the channel (in non-dimensional form) has been plotted for different wedge angles. If can be observed that as the inclination angle increases, the depth of the boundary layer reduces.

\section{Numerical solutions and chaotic behavior}

The Blasius equation (2.16) is solved for different wedge angles to obtain the boundary layer profile. Figure 3 shows the boundary layer depth $\delta$ against the length of the channel for different wedge angles (Eq. 2.13). It can be observed that an increase in the inclination angle results in a reduction of the boundary layer depth.

The system of Eqs. (2.13) is numerically solved for different wedge angles for a given initial perturbation $\left(\delta_{0}=10^{-5}\right)$ at the initial condition and the difference between the two solutions in phase space for the two initial conditions is computed. Figure 4 shows the distance between the two solutions evolved in phase space with an increase in $\eta$. It is observed that as the wedge angle reaches $22^{\circ}$, the difference between the paths in phase space diverges.

The initial guess of the velocity gradient at the surface of the plate is assumed to be unity. The correct velocity gradient has been estimated by solving the Blasius equation using Newton's iterative scheme. In Fig. 5 the velocity gradient value at the plate surface has been plotted against the iteration number. From that figure, it is observed that as the wedge angle increases, the number of iterations required to estimate the velocity gradient at the surface of the wedge increases and when the wedge angle approaches $22^{\circ}$, the solution does not converge.

It can be observed from Table 1 that until $m=0.1335$ $\left(\theta=21.2^{\circ}\right)$, the value of the initial velocity gradient at the surface converges to a unique number, hence the solution of the Blasius equation is consistent. As the value of $m$ crosses 0.1335 the solution becomes periodic with cycle 2 . Hence we do not get a unique solution, but we get a solution periodic in time and space. With increase in $m$, the cycle of periodicity 

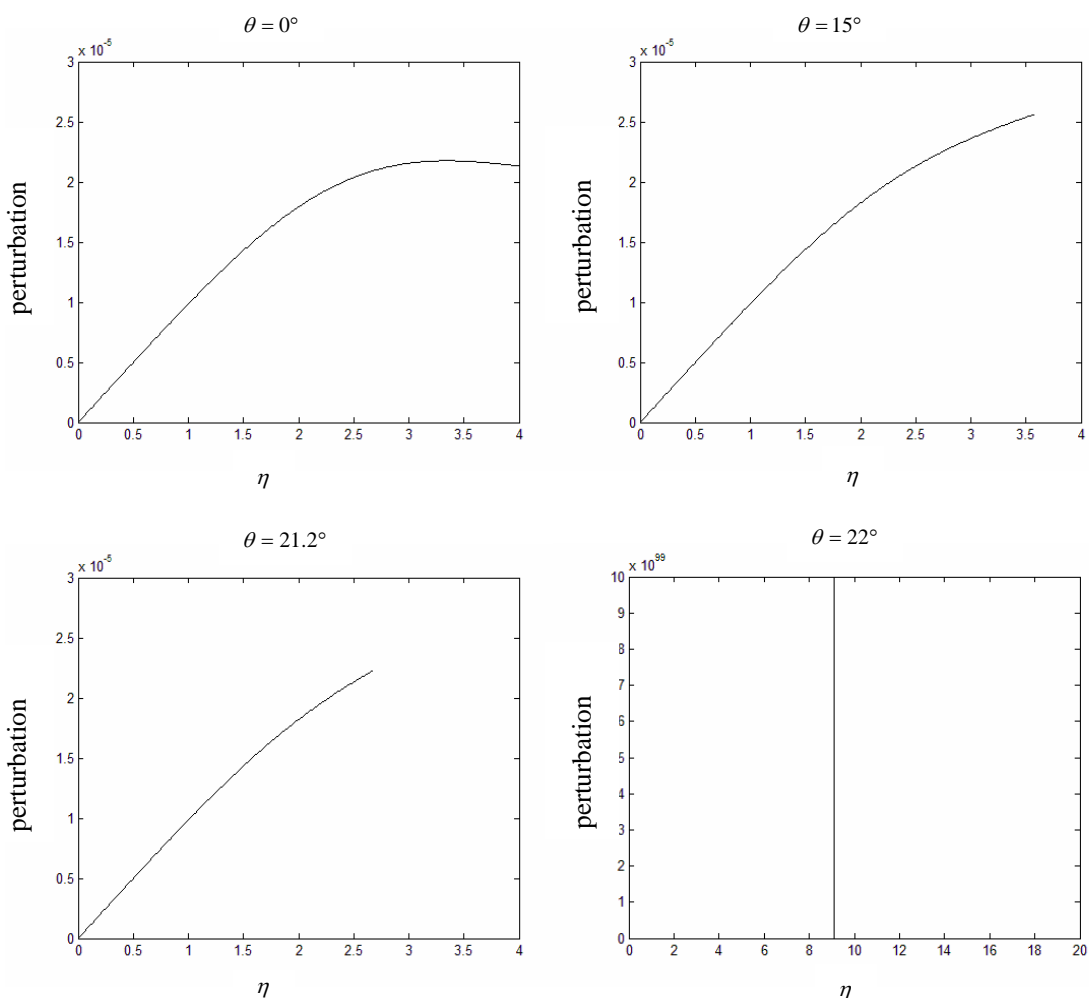

Fig. 4. The difference between the original and perturbed initial conditions is plotted against increase in non-dimensional distance from the plate surface $\eta$. It is observed that at the wedge angle of $22^{\circ}$, the difference between the trajectories in phase space blows up.
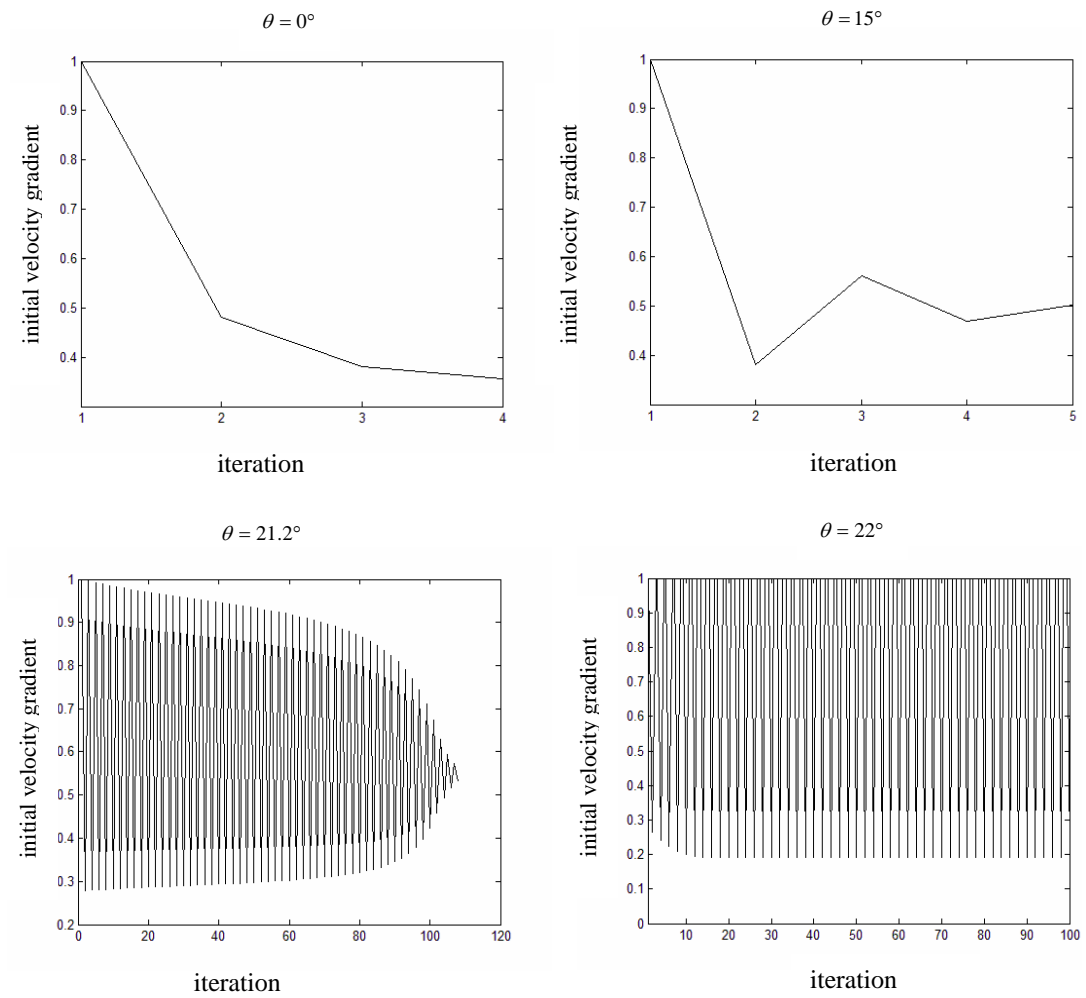

Fig. 5. It is observed that as the wedge angle increases, the number of iterations required to estimate the velocity gradient at the surface of the wedge increases and when the wedge angle is $22^{\circ}$, the solution does not converge. 
Table 1. The estimated initial guess for different wedge angles had been tabulated below. It can be observed that when the angle is $21.3^{\circ}$, the solution begins to oscillate between two values. As we increase the angle further, the method fails to converge to a solution.

\begin{tabular}{lll}
\hline $\begin{array}{l}\theta \\
(\text { degree })\end{array}$ & $m$ & $\begin{array}{l}\text { Initial velocity gradient } \\
(\mathrm{s})\end{array}$ \\
\hline 5 & 0.028571 & 0.3976 \\
10 & 0.0588 & 0.4469 \\
20 & 0.125 & 0.5546 \\
21.2 & 0.1335 & 0.5317 \\
21.3 & 0.1342 & 0.2582 and 1.0843 \\
22.0 & 0.1392 & - \\
\hline
\end{tabular}

increases and eventually at $m=0.1392$, the solution becomes chaotic in nature and the system loses its predictability. To the best of our knowledge, the onset of a chaotic behavior in flow over a wedge has not been reported before in the literature. However, experimental results have shown that for a wedge angle $2 \theta=43.2^{\circ}, \theta=21.6^{\circ}$, the flow starts to create shock waves in the medium (Kewley and Hornung, 1974), which is a representation of chaotic behavior in the flow field. The important question then is, what is the equation that describes the boundary flow dynamics for angles exceeding the critical value of $21.2^{\circ}$. This equation is derived below.

\section{Generalized equation for large wedge angles}

In the derivation of the Blasius equation from the twodimensional Navier-Stokes equation, the velocity components are expressed in the form of the stream-function, selected to result in a similarity solution. This leads to the derivation of the Blasius equation, an ordinary differential equation, from the two-dimensional Navier-Stokes equation, which is a partial differential equation. When the wedge angle is larger than the critical angle and chaotic behavior is observed, the characteristic variables in the partial differential equation cannot be found and hence the stream-function cannot be expressed in terms of a single variable. So, when the wedge angle is greater than the critical angle, the Blasius equation cannot be applied to model the boundary layer phenomenon. Instead, the partial differential equation in terms of the stream-function has to be solved.

Hence for high wedge angle values, the appropriate equation for modeling the boundary layer is Eq. (2.9):

$$
\frac{\partial \psi}{\partial y} \frac{\partial^{2} \psi}{\partial x \partial y}-\frac{\partial \psi}{\partial x} \frac{\partial^{2} \psi}{\partial y^{2}}=u_{0} \frac{\partial u_{0}}{\partial x}+v \frac{\partial^{3} \psi}{\partial y^{3}}
$$

where $u_{0}=U x^{m}$. In this case the model becomes:

$$
\frac{\partial \psi}{\partial y} \frac{\partial^{2} \psi}{\partial x \partial y}-\frac{\partial \psi}{\partial x} \frac{\partial^{2} \psi}{\partial y^{2}}=m U^{2} x^{2 m-1}+v \frac{\partial^{3} \psi}{\partial y^{3}},
$$

with boundary conditions:

$$
\begin{array}{r}
\psi(t, x, 0)=0, \\
\left.\frac{\partial \psi}{\partial y}\right|_{(t, x, 0)}=\frac{u(t, x, 0)}{u_{0}}=0, \\
\left.\frac{\partial \psi}{\partial y}\right|_{(t, x, \infty)}=\frac{u(t, x, \infty)}{u_{0}}=1, \\
-\left.\frac{\partial \psi}{\partial x}\right|_{(t, x, 0)}=\frac{v(t, x, 0)}{u_{0}}=0
\end{array}
$$

This equation can be solved numerically $\forall x$ except at $x=0$ which is the corner of the wedge and a singular point.

Also, depending on the boundary conditions, the flow field may never reach steady state inside the boundary layer. In that case, the time dependent two-dimensional Navier-Stokes equation has to be solved to model the boundary layer, and is given by:

$$
\begin{aligned}
& \frac{\partial u}{\partial x}+\frac{\partial v}{\partial y}=0 \\
& \frac{\partial u}{\partial t}+u \frac{\partial u}{\partial x}+v \frac{\partial u}{\partial y}=u_{0} \frac{\partial u_{0}}{\partial x}+v \frac{\partial^{2} u}{\partial y^{2}}
\end{aligned}
$$

Replacing the velocity components in terms of streamfunction gives:

$u=\frac{\partial \psi}{\partial y}, v=-\frac{\partial \psi}{\partial x}$

and the momentum equation becomes:

$$
\frac{\partial^{2} \psi}{\partial t \partial y}+\frac{\partial \psi}{\partial y} \frac{\partial^{2} \psi}{\partial x \partial y}-\frac{\partial \psi}{\partial x} \frac{\partial^{2} \psi}{\partial y^{2}}=u_{0} \frac{\partial u_{0}}{\partial x}+v \frac{\partial^{3} \psi}{\partial y^{3}}
$$

where $u_{0}$ is expressed as the power law $u_{0}=U x^{m}$. Hence the model becomes:

$$
\frac{\partial^{2} \psi}{\partial t \partial y}+\frac{\partial \psi}{\partial y} \frac{\partial^{2} \psi}{\partial x \partial y}-\frac{\partial \psi}{\partial x} \frac{\partial^{2} \psi}{\partial y^{2}}=m U^{2} x^{2 m-1}+v \frac{\partial^{3} \psi}{\partial y^{3}} .
$$

The boundary conditions are:

$$
\begin{array}{r}
\psi(t, x, 0)=0, \\
\left.\frac{\partial \psi}{\partial y}\right|_{(t, x, 0)}=\frac{u(t, x, 0)}{u_{0}}=0, \\
\left.\frac{\partial \psi}{\partial y}\right|_{(t, x, \infty)}=\frac{u(t, x, \infty)}{u_{0}}=1, \\
-\left.\frac{\partial \psi}{\partial x}\right|_{(t, x, 0)}=\frac{v(t, x, 0)}{u_{0}}=0,
\end{array}
$$

$\left.\frac{\partial \psi}{\partial y}\right|_{(0, x, y)}=\frac{u(0, x, y)}{u_{0}}=1 \quad$ as the flow was uniform

before the presence of the wedge 


\section{Discussion}

The Blasius equation is a third order ordinary differential equation, and thus requires three boundary conditions to obtain its solutions. The values of the stream function and the velocity at the surface of the wedge are two of these boundary conditions. The third boundary condition arises from the fact that the effect of the wedge can be neglected at far distance from the surface. The boundary value problem is converted to an initial value problem by using the third boundary condition. The Blasius equation is solved assuming a velocity gradient and is iterated after every step using Newton-Raphson method until a convergent value of the velocity gradient at the wedge surface is obtained. It is clear from Fig. 5, for $\theta=0^{\circ}$ and $\theta=15^{\circ}$, that the iteration converges rapidly. But for $\theta=21.2^{\circ}$ it takes around 110 iterations to converge. And as the wedge angle reaches $\theta=22^{\circ}$ the iteration fails to converge and starts oscillating. This phenomenon arises when a nonlinear system produces chaotic behavior through bifurcation. To verify chaotic behavior, the Blasius equation is simulated with two nearby initial conditions with a perturbation of $\delta_{0}=10^{-5}$. The equation has been simulated for those two close initial conditions and the distance between the two solutions in the phase space has been plotted in Fig. 4. For $\theta=0^{\circ}$ to $\theta=21.2^{\circ}$, the difference in the two solutions remains bounded, whereas at $\theta=22^{\circ}$, the solution diverges indicating sensitivity to the initial conditions and approach to chaos.

\section{Conclusions}

The Blasius equation is used to model the growth of boundary layer over a flat surface. It has been observed experimentally that as the angle of inclination $\theta$ of the surface with respect to the direction of the flow increases, shock waves are created. Also the analytical solution of the Blasius equation generates multiple solutions when $\theta$ crosses a certain critical value. This led us to analyze the Blasius equation for different values of $\theta$ and we found that bifurcation occurs with an increase in $\theta$, eventually leading to onset of chaos. Hence we conclude that beyond a critical angle of inclination, which is quantified to be $21.2^{\circ}$, the Blasius equation cannot be applied to model the boundary layer growth over a flat inclined surface. Instead, a different equation describes the flow dynamics and in this contribution we provide a derivation of this equation.

\section{Appendix A}

\section{Shooting method using Newton-Raphson method}

Let $h(\eta=0)=s$. Since $s$ is unknown, we assume the value of $s$ and solve the equation accordingly. The boundary condition is: $f(\eta=0)=0, g(\eta=0)=0, g\left(\eta=\eta_{L}\right)=1$. So the value of $s$ should be such that $g\left(\eta=\eta_{L}\right)=1$. Hence we define a function $\chi$ such that:

$\chi\left(s, \eta_{L}\right)=g\left(\eta_{L}\right)-1$ and we solve $s$ for $\chi=0$.

To solve for $s$, we check the sensitivity of the variables $f, g$, and $h$ with respect to $s$. Hence we define three new variables:

$F=\frac{\partial f}{\partial s}, G=\frac{\partial g}{\partial s}, \quad H=\frac{\partial h}{\partial s}$

Now,

$\frac{\partial F}{\partial \eta}=\frac{\partial}{\partial \eta}\left(\frac{\partial f}{\partial s}\right)=\frac{\partial}{\partial s}\left(\frac{\partial f}{\partial \eta}\right)=\frac{\partial g}{\partial s}=G$.

Similarly, $\frac{\partial G}{\partial \eta}=H$

$$
\begin{aligned}
\frac{\partial H}{\partial \eta}=\frac{\partial}{\partial \eta}\left(\frac{\partial h}{\partial s}\right) & =\frac{\partial}{\partial s}\left(-\frac{(m+1)}{2} f h-m\left(1-g^{2}\right)\right) \\
& =-\frac{(m+1)}{2} \frac{\partial}{\partial s}(f h)-m \frac{\partial}{\partial s}\left(1-g^{2}\right) \\
& =-\frac{(m+1)}{2}(F h+f H)+2 m g G
\end{aligned}
$$

Using Newton's method,

$$
\begin{aligned}
s_{i+1} & =s_{i}-\frac{\chi\left(s, \eta_{L}\right)}{\chi^{\prime}\left(s, \eta_{L}\right)}=s_{i}-\frac{\chi\left(s, \eta_{L}\right)}{\left.\frac{\partial \chi\left(s, \eta_{L}\right)}{\partial s}\right|_{\left(s_{i}, \eta_{L}\right)}} \\
& =s_{i}-\frac{g\left(s_{i}, \eta_{L}\right)-1}{\left.\frac{\partial g}{\partial s}\right|_{\left(s_{i}, \eta_{L}\right)}}=s_{i}-\frac{g\left(s_{i}, \eta_{L}\right)-1}{G\left(s_{i}, \eta_{L}\right)}
\end{aligned}
$$

the value of $s$ is obtained by iteration.

Also to solve for $\eta_{L}$, we use the condition that

$\left.\frac{\partial u}{\partial \eta}\right|_{\eta_{L}}=0 \Rightarrow f^{\prime \prime}\left(\eta_{L}\right)=0$

Newton's method can be applied if the system is well defined, continuous, differentiable and where existence and uniqueness theorem holds. If the system becomes sensitive to the initial condition, i.e., if the system becomes chaotic in nature, then slight error in the estimation of $s$ will be enhanced as $\eta$ increases eventually giving highly erroneous results at $\eta_{L}$.

Acknowledgements. The paper was presented at the Chapman Conference on Complexity and Extreme Events, Hyderabad, February 2010, and the authors thank NSF for travel support. The authors also acknowledge partial support by the National Center for Earth-surface Dynamics (NCED) funded by NSF under agreement EAR-0120914 and also the Mathematics in Geosciences NSF grants EAR-0824084 and EAR-0835789.

Edited by: V. P. Dimri

Reviewed by: R. K. Tiwari and another anonymous referee 


\section{References}

Adomian, G.: A review of the decomposition method in applied mathematics, J. Math. Anal. Appl., 135, 501-544, doi:10.1016/0022-247X(88)90170-9, 1988.

Adomian, G.: A review of the decomposition method and some recent results for nonlinear equations, Comput. Math. Appl., 21(2), 101-127, doi:10.1016/0898-1221(91)90220-X, 1991.

Adomian, G.: Solution of physical problems by decomposition, Comput. Math. Appl., 27(9-10), 145-154, doi:10.1016/08981221(94)90132-5, 1994.

Allan, F. M. and Syam, M. I.: On the analytic solutions of the nonhomogeneous Blasius problem, J. Comput. Appl. Math., 182, 362-371, 2005.

Brighi, B. and Hoernel, J. D.: Recent Advances on Similarity Solutions Arising During Free Convection, Prog. Nonlin., 63, 83-92, 2005.

Brighi, B. and Hoernel, J. D.: Similarity solutions for high frequency excitation of liquid metal in an antisymmetric magnetic field, Banach Center Pub., 74, 41-57, 2006.

Brighi, B. and Sari, T.: Blowing-up coordinates for a similarity boundary layer equation, Discret. Contin. Dyn. S. (DCDS-A), 12(5), 929-948, doi:10.3934/dcds.2005.12.929, 2005.

Cortell, R.: Flow and heat transfer in a moving fluid over a moving flat surface, Theor. Comp. Fluid Dyn., 21(6), 435-446, doi:10.1007/s00162-007-0056-z, 2007.

Fang, T.: Further study on a moving-wall boundary-layer problem with mass transfer, Acta Mech., 163(3-4), 183-188, doi:10.1007/s00707-002-0979-9, 2003a.

Fang, T.: Similarity solutions for a moving-flat plate thermal boundary layer, Acta Mech., 163(3-4), 161-172, doi:10.1007/s00707003-0004-y, 2003b.
Hussaini, M. Y. and Lakin, W. D.: Existence and non-uniqueness of similarity solutions of a boundary-layer problem, Q. J. Mech. Appl. Math., 39(1), 15-23, doi:10.1093/qjmam/39.1.15, 1986.

Kewley, D. J. and Hornung, H. G.: Non-equilibrium dissociating nitrogen flow over a wedge, J. Fluid Mech., 64(4), 725-736, 1974.

Klemp, J. P. and Acrivos, A.: A moving-wall boundary layer with reverse flow, J. Fluid Mech., 53(1), 177-191, 1972.

Sakiadis, B. C.: Boundary-layer behavior on continuous solid surface: I. Boundary-layer equations for twodimensional and axisymmetric flow, AIChe J., 7, 26-28, doi:10.1002/aic.690070108, 1961.

Schlichting, H. and Gersten, K: Boundary Layer Theory, 8th rev. edn., McGraw-Hill, New York, 1999.

Schlichting, K. and Bussmann, K.: Exakte Losungen für die laminare Grenzschicht mit Absaugung und Ausblasen, Schriften Deutschen Akademie der Luftfahrtforschung Series B, 7(2), 2569, 1943 (in German).

Schwartz, L. W. and Eley, R. R.: Flow of architectural coatings on complex surfaces theory and experiment, J. Eng. Math., 43, 153171, 2002.

Vajravelu, K. and Mohapatra, R. N.: On fluid dynamic drag reduction in some boundary layer flows, Acta Mech., 81, 59-68, doi:10.1007/BF01174555, 1990.

Weyl, H.: On the differential equation of the simplest boundarylayer problems, Ann. Math., 43, 381-407, 1942.

Zaturska, M. B. and Banks, W. H. H.: A new solution branch of the Falkner-Skan equation, Acta Mech., 152, 197-201, doi:10.1007/BF01176954, 2001. 